Homology, Homotopy and Applications, vol.18(1), 2016, pp.247-263

\title{
MODELS FOR MOCK HOMOTOPY CATEGORIES OF PROJECTIVES
}

\author{
JAMES GILLESPIE
}

(communicated by Donald M. Davis)

\begin{abstract}
Let $R$ be a ring and $\mathrm{Ch}(R)$ the category of chain complexes of $R$-modules. We put an abelian model structure on $\mathrm{Ch}(R)$ whose homotopy category is equivalent to $K(\operatorname{Proj})$, the homotopy category of all complexes of projectives. However, the cofibrant objects are not complexes of projectives, but rather all complexes of flat modules. The trivial objects are what Positselski calls contraacyclic complexes and so the homotopy category coincides with his contraderived category. We in fact construct this model on the category of chain complexes of quasi-coherent sheaves on any scheme $X$ admitting a flat generator. In this case the homotopy category recovers what Murfet calls the mock homotopy category of projectives. In the same way we construct a model for the (mock) projective stable derived category, and we use model category methods to recover the recollement of Murfet. Finally, we consider generalizations by replacing the flat cotorsion pair with other complete hereditary cotorsion pairs in Grothendieck categories.
\end{abstract}

\section{Introduction}

Let $X$ be a separated noetherian scheme and $\mathrm{Qco}(X)$ the category of quasicoherent sheaves on $X$. In Murfet's thesis [17], we saw the introduction of the mock homotopy category of projectives $K_{m}(\operatorname{Proj} X)$. While the category $\mathrm{Qco}(X)$ typically does not have enough projectives, Murfet showed that this triangulated category $K_{m}(\operatorname{Proj} X)$, which is a certain Verdier localization of the homotopy category of flat sheaves, fills the role one would expect from a homotopy category of projectives. In particular, as Neeman originally showed in $[\mathbf{1 8}], K_{m}(\operatorname{Proj} X)$ agrees with the usual homotopy category of projectives in the case that $X$ is affine. And, Murfet's thesis shows in detail how $K_{m}(\operatorname{Proj} X)$ plays a fundamental role in an extension of classical Grothendieck duality.

The author thanks the referee for his or her useful feedback and careful reading of the manuscript. Received January 26, 2015, revised September 3, 2015, October 12, 2015; published on April 27, 2016.

2010 Mathematics Subject Classification: 55U35, 18G25, 18E35.

Key words and phrases: abelian model structure, chain complex of projectives, triangulated category, quasi-coherent sheaf, recollement.

Article available at http://dx.doi.org/10.4310/HHA.2016.v18.n1.a13

Copyright (C) 2016, International Press. Permission to copy for private use granted. 
It is an interesting question whether or not we can describe these phenomena using cotorsion pairs and abelian model categories. Several authors have recently considered this question, for example, see $[\mathbf{6}, \mathbf{2 2}, \mathbf{1 2}]$. In particular, Šťovíček $[\mathbf{2 2}]$ and Gillespie [12] each constructed model structures on the category of complexes of flat sheaves whose homotopy category is equivalent to $K_{m}(\operatorname{Proj} X)$. But the category of complexes of flat sheaves does not have all small limits and colimits and so the resulting exact model structures do not give us a model category in the usual sense. A closer inspection shows that we would like a model structure on the entire chain complex category such that: (1) the cofibrant objects are exactly the complexes of flat quasi-coherent sheaves, and, (2) in the affine case, its homotopy category recovers Positselski's contraderived category (from [19]), which in turn is equivalent to $K(\operatorname{Proj})$, the homotopy category of all complexes of projectives. Analogous to the usual derived category which is obtained as a Verdier quotient by killing the exact (or acyclic) complexes, the contraderived category is the Verdier quotient obtained by killing the contraacyclic complexes. By definition, these are the complexes $W$ such that every chain map $P \rightarrow W$ is null homotopic whenever $P$ is a complex of projectives.

So the first goal of this paper is to construct such a model structure, satisfying the conditions (1) and (2) above. Its homotopy category corresponds exactly to Murfet's mock homotopy category of projectives, $K_{m}(\operatorname{Proj} X)$. In fact, if we restrict the model to the Quillen equivalent model inherited by the fully exact subcategory of cofibrant objects, that is, the exact category of chain complexes of flat sheaves, then the resulting exact model structure recovers the previous models constructed in $[\mathbf{6}, \mathbf{2 2}, \mathbf{1 2}]$. The surprising thing is that the model structure we seek is now quite easy to construct due to a new method for constructing abelian model structures that appeared in [13]. This method works well in situations where we do not know the exact nature of the trivial objects. For example, when looking to construct a model structure for $K_{m}(\operatorname{Proj} X)$, we know that a complex $F$ of flat sheaves ought to be trivial whenever it is exact and has each cycle $Z_{n} F$ flat. But for complexes without flat components, it is not clear (for non-affine schemes) what the trivial complexes should be. How can one construct a model structure when one does not have an explicit description of the trivial objects? The paper [13] provides the answer we need to this question. We apply it in Section 4 to easily obtain, using already known completeness results for cotorsion pairs of complexes, the desired model structure for $K_{m}(\operatorname{Proj} X)$. Using the same method we will obtain a model structure for Murfet's mock projective stable derived category, denoted $K_{m, a c}(\operatorname{Proj} X)$.

But the much more ambitious goal of this paper is to give a model category description of the recollement from Murfet's thesis involving $K_{m}(\operatorname{Proj} X), K_{m, a c}(\operatorname{Proj} X)$, and the usual derived category $\mathcal{D}(X)$. This recollement is dual to the injective recollement of Krause which was interpreted via model category methods by Becker in [1]. This method was generalized in [11], and in the current paper we see in Theorems 3.5 and 3.6 similar, but more general results, allowing one to obtain recollement situations from abelian model structures. This method is purely (model) categorical and by stripping away all of the algebraic geometry we see that we can obtain the recollement whenever $X$ is a scheme for which $\mathrm{Qco}(X)$ admits a flat generator. For example, this is true whenever $X$ is a quasi-compact and semi-separated scheme. In fact the method is so general that we show in the last section how to obtain a similar 
recollement by starting with any hereditary cotorsion pair $(\mathcal{A}, \mathcal{B})$ in a Grothendieck category $\mathcal{G}$, as long as it is cogenerated by a set $\mathcal{S}$ and $\mathcal{A}$ contains a generating set $\left\{U_{i}\right\}$ for $\mathcal{G}$.

\section{Notation and preliminaries}

For the convenience of the reader we summarize some essential definitions in this section. This includes cotorsion pairs and abelian model structures, recollement situations, and deconstructibility. Throughout this section and Section 3 we let $\mathcal{A}$ denote a fixed abelian category.

\subsection{Cotorsion pairs and abelian model structures}

Hovey showed in $[\mathbf{1 5}$ ] that an abelian model category $\mathcal{A}$ is nothing more than two nicely related cotorsion pairs in $\mathcal{A}$. By definition, a pair of classes $(\mathcal{X}, \mathcal{Y})$ in $\mathcal{A}$ is called a cotorsion pair if $\mathcal{Y}=\mathcal{X}^{\perp}$ and $\mathcal{X}={ }^{\perp} \mathcal{Y}$. Here, given a class of objects $\mathcal{C}$ in $\mathcal{A}$, the right orthogonal $\mathcal{C}^{\perp}$ is defined to be the class of all objects $X$ such that $\operatorname{Ext}_{\mathcal{A}}^{1}(C, X)=0$ for all $C \in \mathcal{C}$. Similarly, we define the left orthogonal ${ }^{\perp} \mathcal{C}$. We call $\mathcal{X} \cap \mathcal{Y}$ the core of the cotorsion pair, and we call a cotorsion pair hereditary if $\operatorname{Ext}_{\mathcal{A}}^{i}(X, Y)=0$ for all $X \in \mathcal{X}, Y \in \mathcal{Y}$, and $i \geqslant 1$. The cotorsion pair is complete if it has enough injectives and enough projectives. This means that for each $A \in \mathcal{A}$ there exist short exact sequences $0 \rightarrow A \rightarrow Y \rightarrow X \rightarrow 0$ and $0 \rightarrow Y^{\prime} \rightarrow X^{\prime} \rightarrow A \rightarrow 0$ with $X, X^{\prime} \in \mathcal{X}$ and $Y, Y^{\prime} \in \mathcal{Y}$. Besides their connection to abelian model structures which we describe next, cotorsion pairs are fundamental in modern homological algebra. There are several good references. In particular we will refer to [5] and [15].

The main theorem of [15] showed that an abelian model structure on $\mathcal{A}$ is equivalent to a triple $(\mathcal{C}, \mathcal{W}, \mathcal{F})$ of classes of objects in $\mathcal{A}$ for which $\mathcal{W}$ is thick and $(\mathcal{C} \cap \mathcal{W}, \mathcal{F})$ and $(\mathcal{C}, \mathcal{W} \cap \mathcal{F})$ are each complete cotorsion pairs. By thick we mean that the class $\mathcal{W}$ is closed under retracts and satisfies that whenever two out of three terms in a short exact sequence are in $\mathcal{W}$ then so is the third. In this case, $\mathcal{C}$ is precisely the class of cofibrant objects of the model structure, $\mathcal{F}$ are precisely the fibrant objects, and $\mathcal{W}$ is the class of trivial objects. We hence denote an abelian model structure $\mathcal{M}$ as a triple $\mathcal{M}=(\mathcal{C}, \mathcal{W}, \mathcal{F})$ and for short we will denote the two associated cotorsion pairs above by $(\widetilde{\mathcal{C}}, \mathcal{F})$ and $(\mathcal{C}, \widetilde{\mathcal{F}})$, and often refer to $\mathcal{M}$ as a Hovey triple. We say that $\mathcal{M}$ is hereditary if both of the associated cotorsion pairs are hereditary. Finally, by the core of an abelian model structure $\mathcal{M}=(\mathcal{C}, \mathcal{W}, \mathcal{F})$ we mean the class $\mathcal{C} \cap \mathcal{W} \cap \mathcal{F}$. Note that it coincides with the cores of both $(\widetilde{\mathcal{C}}, \mathcal{F})$ and $(\mathcal{C}, \widetilde{\mathcal{F}})$.

Given any Hovey triple $\mathcal{M}=(\mathcal{C}, \mathcal{W}, \mathcal{F})$ there are four approximation sequences associated to $\mathcal{M}$. In particular, using enough injectives of $(\widetilde{\mathcal{C}}, \mathcal{F})$ corresponds to the fibrant replacement functor denoted by $R$. On the other hand, using enough projectives of $(\mathcal{C}, \widetilde{\mathcal{F}})$ corresponds to the cofibrant replacement functor which we denote by $Q$. However, by using enough projectives of $(\widetilde{\mathcal{C}}, \mathcal{F})$ we also get a functor which we denote by $\widetilde{Q}$ and by using enough injectives of $(\mathcal{C}, \widetilde{\mathcal{F}})$ we get a functor we denote by $\widetilde{R}$. When we encounter multiple abelian model structures we use subscripts such as $\mathcal{M}_{1}, \mathcal{M}_{2}, \mathcal{M}_{3}$ and denote these associated functors with notations such as $R_{2}, \widetilde{Q}_{1}$, $\widetilde{R}_{3}$, etc.

A recent result appearing in $[\mathbf{1 3}]$ will prove fundamental to this paper. It says 
that whenever $(\widetilde{\mathcal{C}}, \mathcal{F})$ and $(\mathcal{C}, \widetilde{\mathcal{F}})$ are complete hereditary cotorsion pairs with equal cores and $\widetilde{\mathcal{F}} \subseteq \mathcal{F}$, then there is a unique thick class $\mathcal{W}$ yielding a Hovey triple $\mathcal{M}=$ $(\mathcal{C}, \mathcal{W}, \mathcal{F})$ with $\mathcal{C} \cap \mathcal{W}=\widetilde{\mathcal{C}}$ and $\mathcal{W} \cap \mathcal{F}=\widetilde{\mathcal{F}}$. Besides [15] we will refer to [14] for any other basics from the theory of model categories.

\subsection{Recollement situations}

The homotopy category of an hereditary abelian model category is always a triangulated category. See [10, Prop. 5.2(4)/Cor. 5.4] and [1, Prop. 1.1.14/Cor. 1.1.15]. Quillen functors between such categories naturally give rise to left derived functors, in particular left adjoints, between these associated triangulated homotopy categories. This paper aims to describe recollements of triangulated categories using Quillen functors. Loosely, a recollement is an "attachment" of two triangulated categories described in terms of adjunctions satisfying strong exactness conditions. The standard reference is [2], although the definitions below will surely suffice for the reader.

Definition 2.1. Let $\mathcal{T}^{\prime} \stackrel{F}{\rightarrow} \mathcal{T} \stackrel{G}{\rightarrow} \mathcal{T}^{\prime \prime}$ be a sequence of exact functors between triangulated categories. We say it is a localization sequence when there exists right adjoints $F_{\rho}$ and $G_{\rho}$ giving a diagram of functors

$$
\mathcal{T}^{\prime} \underset{F_{\rho}}{\stackrel{F}{\rightleftarrows}} \mathcal{T} \underset{G_{\rho}}{\stackrel{G}{\rightleftarrows}} \mathcal{T}^{\prime \prime}
$$

with the listed properties:

1. The right adjoint $F_{\rho}$ of $F$ satisfies $F_{\rho} \circ F \cong \operatorname{id}_{\mathcal{T}^{\prime}}$.

2. The right adjoint $G_{\rho}$ of $G$ satisfies $G \circ G_{\rho} \cong \mathrm{id}_{\mathcal{T}^{\prime \prime}}$.

3. For any object $X \in \mathcal{T}$, we have $G X=0$ iff $X \cong F X^{\prime}$ for some $X^{\prime} \in \mathcal{T}^{\prime}$.

The notion of a colocalization sequence is the dual. That is, there must exist left adjoints $F_{\lambda}$ and $G_{\lambda}$ with the analogous properties.

Note the similarity in the definitions above to the notion of a split exact sequence, but for adjunctions. It is true that if $\mathcal{T}^{\prime} \stackrel{F}{\rightarrow} \mathcal{T} \stackrel{G}{\rightarrow} \mathcal{T}^{\prime \prime}$ is a localization sequence then $\mathcal{T}^{\prime \prime} \stackrel{G_{\rho}}{\longrightarrow} \mathcal{T} \stackrel{F_{\rho}}{\rightarrow} \mathcal{T}^{\prime}$ is a colocalization sequence and if $\mathcal{T}^{\prime} \stackrel{F}{\rightarrow} \mathcal{T} \stackrel{G}{\rightarrow} \mathcal{T}^{\prime \prime}$ is a colocalization sequence then $\mathcal{T}^{\prime \prime} \stackrel{G_{\lambda}}{\longrightarrow} \mathcal{T} \stackrel{F_{\lambda}}{\longrightarrow} \mathcal{T}^{\prime}$ is a localization sequence. This brings us to the definition of a recollement where the sequence of functors $\mathcal{T}^{\prime} \stackrel{F}{\rightarrow} \mathcal{T} \stackrel{G}{\rightarrow} \mathcal{T}^{\prime \prime}$ is both a localization sequence and a colocalization sequence.

Definition 2.2. Let $\mathcal{T}^{\prime} \stackrel{F}{\rightarrow} \mathcal{T} \stackrel{G}{\rightarrow} \mathcal{T}^{\prime \prime}$ be a sequence of exact functors between triangulated categories. We say $\mathcal{T}^{\prime} \stackrel{F}{\rightarrow} \mathcal{T} \stackrel{G}{\rightarrow} \mathcal{T}^{\prime \prime}$ induces a recollement if it is both a localization sequence and a colocalization sequence as shown in the picture.

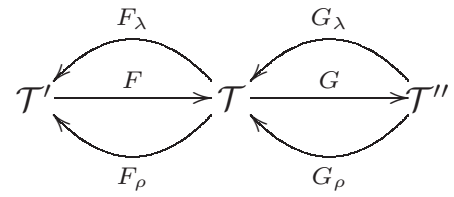

So the idea is that a recollement is a colocalization sequence "glued" with a localization sequence. 


\subsection{Deconstructibility}

In applications the abelian categories we work with are actually Grothendieck categories and in the final Section 4.1 we will use the notion of deconstructibility in Grothendieck categories. This concept has been nicely developed by Šťovíček in [21].

Definition 2.3. A class of objects $\mathcal{F}$ is called deconstructible if $\mathcal{F}=$ Filt- $\mathcal{S}$ for some set $\mathcal{S}$. Here Filt- $\mathcal{S}$ denotes the class of all transfinite extensions of $\mathcal{S}$. So in particular, $\mathcal{S} \subseteq \mathcal{F}$ and $\mathcal{F}$ is the class of all transfinite extensions of $\mathcal{S}$.

By a transfinite extension of $\mathcal{S}$ we mean a colimit colim $X_{\alpha}$, where $X: \lambda \rightarrow \mathcal{G}$ is a colimit-preserving functor whose domain is an ordinal $\lambda$ and satisfying the following: $X_{0}=0$ and each map $X_{\alpha} \rightarrow X_{\alpha+1}$ is a monomorphism with cokernel in $\mathcal{S}$.

We then have the following very useful proposition.

Proposition 2.4. Let $\mathcal{G}$ be a Grothendieck category and $\mathcal{X}$ a class of objects. Set $\mathcal{Y}=\mathcal{X}^{\perp}$. Then the following are equivalent:

1. $\mathcal{X}$ is deconstructible, closed under retracts, and contains a generating set $\left\{U_{i}\right\}$ for $\mathcal{G}$.

2. $(\mathcal{X}, \mathcal{Y})$ is a complete cotorsion pair cogenerated by some set $\mathcal{S}$.

3. $(\mathcal{X}, \mathcal{Y})$ is a cotorsion pair cogenerated by some set $\mathcal{S}$ and $\mathcal{X}$ contains a generating set $\left\{U_{i}\right\}$ for $\mathcal{G}$.

Moreover, in case (3), every object in $\mathcal{X}$ is a retract of a transfinite extension of objects in $\mathcal{S} \cup\left\{U_{i}\right\}$.

Proof. We first show (1) implies (2). So assume we have some set $\mathcal{S}^{\prime}$ such that $\mathcal{X}=$ Filt- $\mathcal{S}^{\prime}$ is deconstructible. We note that by [21, Lemma 1.6] $\mathcal{X}$ itself must be closed under transfinite extensions. Set $\mathcal{S}=\mathcal{S}^{\prime} \cup\left\{U_{i}\right\}$. Then by [20, Corollary 2.14 (2)] $\mathcal{S}$ cogenerates a complete cotorsion pair $\left({ }^{\perp}\left(\mathcal{S}^{\perp}\right), \mathcal{S}^{\perp}\right)$ and ${ }^{\perp}\left(\mathcal{S}^{\perp}\right)$ consists precisely of retracts of transfinite extensions of objects in $\mathcal{S}$. But since $\mathcal{X}$ is closed under transfinite extensions, retracts, and contains $\left\{U_{i}\right\}$, it follows that ${ }^{\perp}\left(\mathcal{S}^{\perp}\right)=\mathcal{X}$.

For the converse, suppose $(\mathcal{X}, \mathcal{Y})$ is a complete cotorsion pair cogenerated by a set $\mathcal{S}$, meaning $\mathcal{S}^{\perp}=\mathcal{Y}$. If $\left\{U_{i}\right\}$ is any generating set for $\mathcal{G}$ and if for each $i$ we have an epimorphism $X_{i} \rightarrow U_{i}$ with $X_{i} \in \mathcal{X}$, then $\left\{X_{i}\right\}$ too is a generating set. So since the cotorsion pair is complete we can always find a generating set $\left\{U_{i}\right\}$ contained in $\mathcal{X}$. It is easy to see that $\mathcal{X}$ is closed under retracts, so it is left to show it is deconstructible. We cannot expect to have $\mathcal{X}=$ Filt- $\mathcal{S}$; we must replace $\mathcal{S}$ with another set $\mathcal{S}^{\prime}$. We start by considering $\mathcal{T}=\mathcal{S} \cup\left\{U_{i}\right\}$. Then still $\mathcal{T}^{\perp}=\mathcal{Y}$ because $\left\{U_{i}\right\} \subseteq \mathcal{X}$. So now $\left({ }^{\perp}\left(\mathcal{T}^{\perp}\right), \mathcal{T}^{\perp}\right)=\left({ }^{\perp}\left(\mathcal{S}^{\perp}\right), \mathcal{S}^{\perp}\right)=(\mathcal{X}, \mathcal{Y})$ and again by [20, Corollary $\left.2.14(2)\right]$ we conclude that $\mathcal{X}$ consists precisely of retracts of objects in the deconstructible class Filt- $\mathcal{T}$. But now [21, Proposition 2.9(1)] tells us that $\mathcal{X}=$ Filt- $\mathcal{S}^{\prime}$ for some set $\mathcal{S}^{\prime}$. So (2) implies (1), and the above arguments also make it clear that (2) and (3) are equivalent.

\section{Localization and recollement via model categories}

Our main goal is to prove Theorems 3.5 and 3.6 which automatically produce a recollement from three interrelated Hovey triples. 
Proposition 3.1. Let $\mathcal{M}_{1}=\left(\mathcal{C}, \mathcal{W}_{1}, \mathcal{F}_{1}\right)$ and $\mathcal{M}_{2}=\left(\mathcal{C}, \mathcal{W}_{2}, \mathcal{F}_{2}\right)$ be hereditary abelian model structures with equal cores $\mathcal{C} \cap \mathcal{W}_{1} \cap \mathcal{F}_{1}=\mathcal{C} \cap \mathcal{W}_{2} \cap \mathcal{F}_{2}$, and $\mathcal{F}_{2} \subseteq \mathcal{F}_{1}$. Then there exists an hereditary abelian model structure $\mathcal{M}_{1} / \mathcal{M}_{2}=\left(\widetilde{\mathcal{C}}_{2}, \mathcal{W}, \mathcal{F}_{1}\right)$, called the right localization of $\mathcal{M}_{1}$ with respect to $\mathcal{M}_{2}$. Here

$$
\begin{aligned}
\mathcal{W} & =\left\{X \in \mathcal{A} \mid \exists \text { a short exact sequence } X \mapsto F_{2} \rightarrow C_{1} \text { with } F_{2} \in \mathcal{F}_{2}, C_{1} \in \widetilde{\mathcal{C}}_{1}\right\} \\
& =\left\{X \in \mathcal{A} \mid \exists \text { a short exact sequence } F_{2}^{\prime} \mapsto C_{1}^{\prime} \rightarrow X \text { with } F_{2}^{\prime} \in \mathcal{F}_{2}, C_{1}^{\prime} \in \widetilde{\mathcal{C}}_{1}\right\} .
\end{aligned}
$$

Proof. We have the two complete hereditary cotorsion pairs $\left(\widetilde{\mathcal{C}}_{1}, \mathcal{F}_{1}\right)$ and $\left(\widetilde{\mathcal{C}}_{2}, \mathcal{F}_{2}\right)$ satisfying $\mathcal{F}_{2} \subseteq \mathcal{F}_{1}$ and $\widetilde{\mathcal{C}}_{1} \cap \mathcal{F}_{1}=\widetilde{\mathcal{C}}_{2} \cap \mathcal{F}_{2}$. Applying [13, Theorem 1.1] we immediately obtain a unique thick class $\mathcal{W}$, with the two descriptions above, making $\left(\widetilde{\mathcal{C}}_{2}, \mathcal{W}, \mathcal{F}_{1}\right)$ an hereditary abelian model structure.

We note that the above proposition is a generalization of a recent result of Becker from [1]. The same is true for the next proposition. For the statement, recall from Section 2.1 the notation we are using for the functors $R, \widetilde{R}, Q$, and $\widetilde{Q}$ associated to an abelian model structure $\mathcal{M}$.

Proposition 3.2. Let $\mathcal{M}_{1}=\left(\mathcal{C}, \mathcal{W}_{1}, \mathcal{F}_{1}\right)$ and $\mathcal{M}_{2}=\left(\mathcal{C}, \mathcal{W}_{2}, \mathcal{F}_{2}\right)$ be hereditary abelian model structures with equal cores and $\mathcal{F}_{2} \subseteq \mathcal{F}_{1}$. Then we have a colocalization sequence $\mathrm{Ho}\left(\mathcal{M}_{2}\right) \stackrel{R(\mathrm{Id})=R_{2}}{\longrightarrow} \mathrm{Ho}\left(\mathcal{M}_{1}\right) \stackrel{R(\mathrm{Id})=R_{1}}{\longrightarrow} \mathrm{Ho}\left(\mathcal{M}_{1} / \mathcal{M}_{2}\right)$ with left adjoints $Q_{1}$ and $\widetilde{Q}_{2}$ as shown below. Here the vertical arrows are the standard equivalences passing between the homotopy categories and the equivalent homotopy categories of fibrant-cofibrant objects.

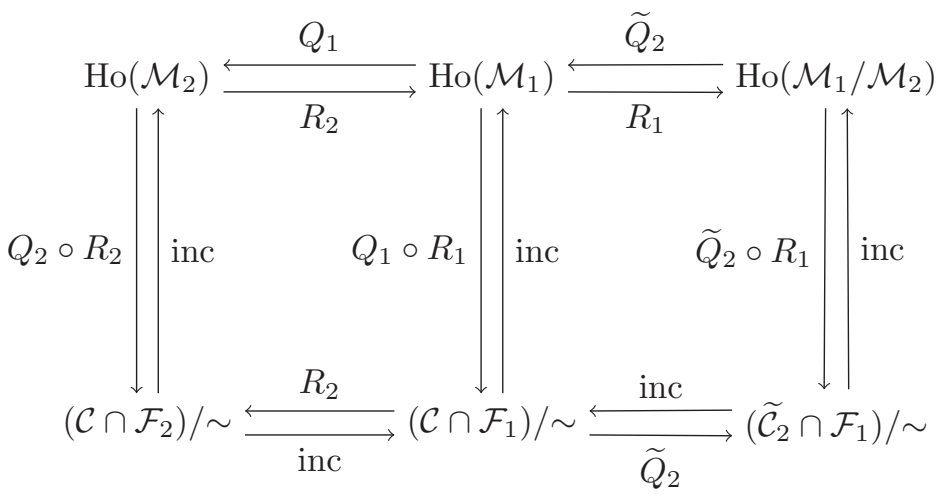

Above we followed a convention that we will use throughout the paper whenever we write functor diagrams: No matter which direction functors are going, we will always write left adjoints on the top or the left, while the right adjoints will be written on the bottom or right.

Proof. The identity functors $\mathcal{M}_{2} \stackrel{\mathrm{Id}}{\rightarrow} \mathcal{M}_{1} \stackrel{\mathrm{Id}}{\rightarrow} \mathcal{M}_{1} / \mathcal{M}_{2}$ are right Quillen functors since they preserve fibrant and trivially fibrant objects. Using the definitions of left and right derived functors we compute the derived adjunctions to be $(L(\mathrm{Id}), R(\mathrm{Id}))=$ $\left(Q_{1}, R_{2}\right)$ and $(L(\mathrm{Id}), R(\mathrm{Id}))=\left(\widetilde{Q}_{2}, R_{1}\right)$. This at least gives us adjunctions as depicted in the top of the diagram. The vertical equivalences passing to the classical stable 
categories are in fact triangle equivalences; see [1, Prop. 1.1.14] and [10, Prop. 5.2/ Cor. 5.4]. Indeed the cited results imply that the full subcategories of fibrant-cofibrant subobjects form a Frobenius category in all three cases. The projective-injective objects in all three cases are the objects belonging to the common core of the three model structures. Considering this we can now see why these functors are exact in the sense that they send exact triangles to exact triangles. We see that when passing to the bottom row of the diagram, each functor in question translates to either an inclusion, or to using enough projectives or injectives with one of the complete hereditary cotorsion pairs in sight. So we wish to show that these functors preserve exact triangles. Now every exact triangle is by definition isomorphic to a standard triangle of the form $X \stackrel{f}{\rightarrow} Y \rightarrow C_{f} \rightarrow \Sigma X$, arising from a short exact sequence $0 \rightarrow$ $X \stackrel{f}{\rightarrow} Y \rightarrow Z \rightarrow 0$ as in the pushout diagram below where $W$ is a projective-injective in the common core of the Hovey triples:

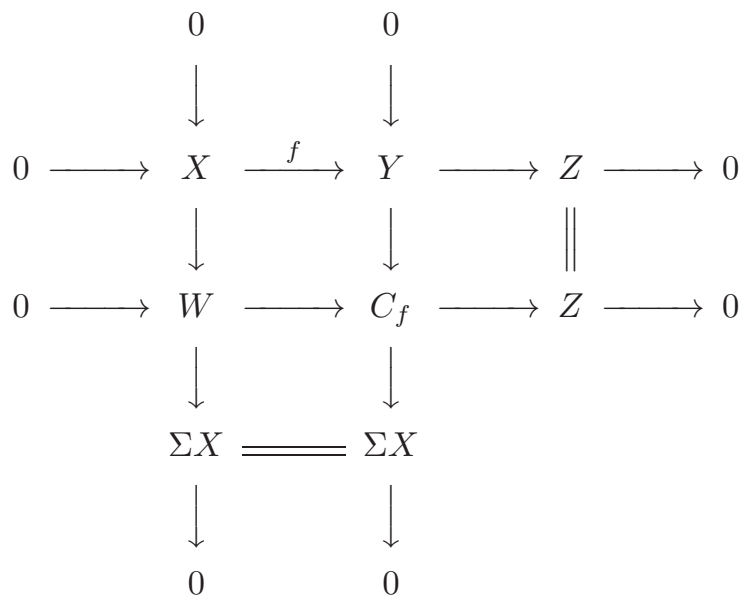

The main point is that by using [1, Lemma 1.4.4 and its dual], along with the fact that all the cotorsion pairs in sight are hereditary with equal cores, any diagram of this form is sent to another diagram of this form. In means that standard triangles (and hence all exact triangles) are sent to standard triangles (resp. exact triangles).

Now to show that the top row is a colocalization sequence, it remains to show:

1. $Q_{1} \circ R_{2} \cong 1_{\mathrm{Ho}\left(\mathcal{M}_{2}\right)}$.

2. $R_{1} \circ \widetilde{Q}_{2} \cong 1_{\mathrm{Ho}\left(\mathcal{M}_{1} / \mathcal{M}_{2}\right)}$.

3. The essential image of $R_{2}$ equals the kernel of $R_{1}$.

To prove (1), let $f: A \rightarrow B$ be a morphism in $\mathcal{A}$. The functor $R_{2}$ acts by $f \mapsto \hat{f}$ where $\hat{f}$ is any map making the diagram below commute. Here the rows are exact, $F_{2}, F_{2}^{\prime} \in \mathcal{F}_{2}$ and $C_{2}, C_{2}^{\prime} \in \widetilde{\mathcal{C}}_{2}=\mathcal{C} \cap \mathcal{W}_{2}$.

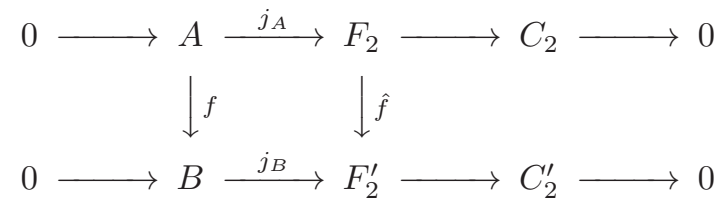


Now applying $Q_{1}$ to $\hat{f}$ gives us $\bar{f}$ in the next commutative diagram. Here $F_{1}, F_{1}^{\prime} \in$ $\widetilde{\mathcal{F}}_{1}=\mathcal{W}_{1} \cap \mathcal{F}_{1}$ and $C, C^{\prime} \in \mathcal{C}$.

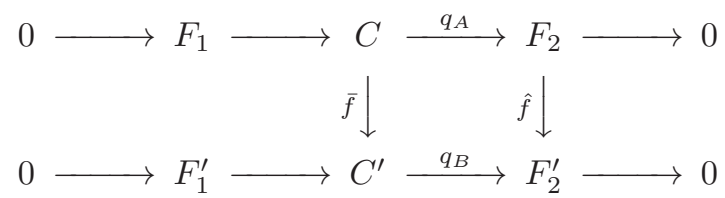

By Lemma 3.3 below we have $\mathcal{W}_{1} \subseteq \mathcal{W}_{2}$. Thus $j_{A}, j_{B}, q_{A}, q_{B}$ are all weak equivalences in $\mathcal{M}_{2}$. So, in $\operatorname{Ho}\left(\mathcal{M}_{2}\right)$, we have a commutative diagram

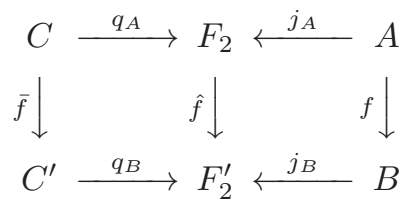

giving rise to a natural isomorphism $\left\{j_{A}^{-1} \circ q_{A}\right\}: Q_{1} \circ R_{2} \cong 1_{\mathrm{Ho}\left(\mathcal{M}_{2}\right)}$.

A similar type of argument to the above will prove (2).

For (3), we start by claiming $\operatorname{ker} R_{1}=\mathcal{W}$, where $\mathcal{W}$ is the class of trivial objects in $\mathcal{M}_{1} / \mathcal{M}_{2}$; so they are precisely the zero objects in $\operatorname{Ho}\left(\mathcal{M}_{1} / \mathcal{M}_{2}\right)$. ( $\subseteq$ ) Say $R_{1} X \in \mathcal{W}$. This means that the fibrant replacement $R_{1} X=F_{1}$ obtained by finding a short exact sequence $0 \rightarrow X \rightarrow F_{1} \rightarrow C_{1} \rightarrow 0$ with $F_{1} \in \mathcal{F}_{1}$ and $C_{1} \in \widetilde{\mathcal{C}}_{1}$, actually has $F_{1} \in$ $\mathcal{W} \cap \mathcal{F}_{1}=\mathcal{F}_{2}$. So by the definition of the class $\mathcal{W}$ in the statement of Proposition 3.1, we have $X \in \mathcal{W}$. () Let $X \in \mathcal{W}$. Then computing $R_{1} X$ with a short exact sequence as we just did, we conclude $R_{1} X \in \mathcal{F}_{2}=\mathcal{W} \cap \mathcal{F}_{1}$. In particular, $R_{1} X=0$ in $\operatorname{Ho}\left(\mathcal{M}_{1} / \mathcal{M}_{2}\right)$.

We proceed to finish the proof of (3). It is clear that the literal image of $R_{2}: \operatorname{Ho}\left(\mathcal{M}_{2}\right) \rightarrow \operatorname{Ho}\left(\mathcal{M}_{1}\right)$ is the class $\mathcal{F}_{2}=\mathcal{W} \cap \mathcal{F}_{1}$, which is contained in ker $R_{1}$. By definition, the essential image of $R_{2}$ is therefore the class of all $X$ that are isomorphic, in $\operatorname{Ho}\left(\mathcal{M}_{1}\right)$, to an object of $\mathcal{F}_{2}$. But it is easy to see that the kernel of any additive functor is closed under isomorphic closure. So ker $R_{1}$ must contain the essential image of $R_{2}$. On the other hand, if $W \in \mathcal{W}=\operatorname{ker} R_{1}$, we have a short exact sequence $0 \rightarrow W \rightarrow F_{2} \rightarrow C_{1} \rightarrow 0$ with $F_{2} \in \mathcal{F}_{2}$ and $C_{1} \in \widetilde{\mathcal{C}}_{1}=\widetilde{\mathcal{C}}_{2} \cap \mathcal{W}$. So $W \rightarrow F_{2}$ is a weak equivalence in $\mathcal{M}_{1} / \mathcal{M}_{2}$ and we are done.

The proof of the above relies on the following easy lemma. It is also crucial to the lemma following it.

Lemma 3.3. Let $\mathcal{M}=(\mathcal{C}, \mathcal{W}, \mathcal{F})$ and $\mathcal{M}^{\prime}=\left(\mathcal{C}, \mathcal{W}^{\prime}, \mathcal{F}^{\prime}\right)$ be abelian model structures having the same cofibrant objects and $\mathcal{F}^{\prime} \subseteq \mathcal{F}$. Then we have $\mathcal{W} \subseteq \mathcal{W}^{\prime}$.

Proof. Since $\mathcal{F}^{\prime} \subseteq \mathcal{F}$, we have a containment of trivially cofibrant objects $\mathcal{C} \cap \mathcal{W} \subseteq$ $\mathcal{C} \cap \mathcal{W}^{\prime}$. Similarly, since the two model structures share the same cofibrant objects we have equality of trivially fibrant objects $\mathcal{W} \cap \mathcal{F}=\mathcal{W}^{\prime} \cap \mathcal{F}^{\prime}$. Now let $W \in \mathcal{W}$. We need to see why $W \in \mathcal{W}^{\prime}$. We write a short exact sequence $0 \rightarrow W^{\prime} \rightarrow C \rightarrow W \rightarrow 0$ with $C \in \mathcal{C}$ and $W^{\prime} \in \mathcal{W}^{\prime} \cap \mathcal{F}^{\prime}$. Then $W^{\prime}$ must also be in $\mathcal{W}$, and since $\mathcal{W}$ is thick, we see that $C \in \mathcal{C} \cap \mathcal{W} \subseteq \mathcal{C} \cap \mathcal{W}^{\prime}$. Since $\mathcal{W}^{\prime}$ is also thick we get $W \in \mathcal{W}^{\prime}$.

For an abelian model structure $\mathcal{M}=(\mathcal{C}, \mathcal{W}, \mathcal{F})$ on $\mathcal{A}$, we let $\gamma: \mathcal{A} \rightarrow \operatorname{Ho}(\mathcal{M})$ denote the canonical localization functor. 
Lemma 3.4. Whenever we have two abelian model structures $\mathcal{M}=(\mathcal{C}, \mathcal{W}, \mathcal{F})$ and $\mathcal{M}^{\prime}=\left(\mathcal{C}^{\prime}, \mathcal{W}^{\prime}, \mathcal{F}^{\prime}\right)$ on $\mathcal{A}$ with $\mathcal{W} \subseteq \mathcal{W}^{\prime}$, then we have a canonical quotient functor $\bar{\gamma}: \operatorname{Ho}(\mathcal{M}) \rightarrow \operatorname{Ho}\left(\mathcal{M}^{\prime}\right)$ for which $\gamma^{\prime}=\bar{\gamma} \circ \gamma$. In particular, if $\mathcal{M}=(\mathcal{C}, \mathcal{W}, \mathcal{F})$ and $\mathcal{M}^{\prime}=\left(\mathcal{C}, \mathcal{W}^{\prime}, \mathcal{F}^{\prime}\right)$ have the same cofibrant objects and $\mathcal{F}^{\prime} \subseteq \mathcal{F}$, then by Lemma 3.3 we have $\bar{\gamma}: \operatorname{Ho}(\mathcal{M}) \rightarrow \operatorname{Ho}\left(\mathcal{M}^{\prime}\right)$.

Proof. In any abelian model structure a map is a weak equivalence if and only if it factors as a monomorphism with trivial cokernel followed by an epimorphism with trivial kernel. So if $\mathcal{W} \subseteq \mathcal{W}^{\prime}$, then the localization functor $\gamma^{\prime}: \mathcal{A} \rightarrow \operatorname{Ho}\left(\mathcal{M}^{\prime}\right)$ sends weak equivalences in $\mathcal{M}$ to isomorphisms. So the universal property of $\gamma$ guarantees the unique functor $\bar{\gamma}: \operatorname{Ho}(\mathcal{M}) \rightarrow \operatorname{Ho}\left(\mathcal{M}^{\prime}\right)$ for which $\gamma^{\prime}=\bar{\gamma} \circ \gamma$.

Theorem 3.5 (Right recollement theorem). Let $\mathcal{A}$ be an abelian category with three hereditary model structures, as below, whose cores all coincide:

$$
\mathcal{M}_{1}=\left(\mathcal{C}, \mathcal{W}_{1}, \mathcal{F}_{1}\right), \quad \mathcal{M}_{2}=\left(\mathcal{C}, \mathcal{W}_{2}, \mathcal{F}_{2}\right), \quad \mathcal{M}_{3}=\left(\mathcal{C}, \mathcal{W}_{3}, \mathcal{F}_{3}\right)
$$

If $\mathcal{W}_{3} \cap \mathcal{F}_{1}=\mathcal{F}_{2}$ and $\mathcal{F}_{3} \subseteq \mathcal{F}_{1}$ (or equivalently, $\widetilde{\mathcal{C}}_{2} \cap \mathcal{W}_{3}=\widetilde{\mathcal{C}}_{1}$ and $\mathcal{F}_{2} \subseteq \mathcal{W}_{3}$ ), then $\operatorname{Ho}\left(\mathcal{M}_{1} / \mathcal{M}_{2}\right)=\operatorname{Ho}\left(\mathcal{M}_{3}\right)$ and $\operatorname{Ho}\left(\mathcal{M}_{1} / \mathcal{M}_{3}\right) \cong \operatorname{Ho}\left(\mathcal{M}_{2}\right)$. In fact, $\mathcal{M}_{1} / \mathcal{M}_{3}$ is Quillen equivalent to $\mathcal{M}_{2}$, while $\mathcal{M}_{1} / \mathcal{M}_{2}$ is Quillen equivalent to $\mathcal{M}_{3}$, and we even have a recollement as shown below.

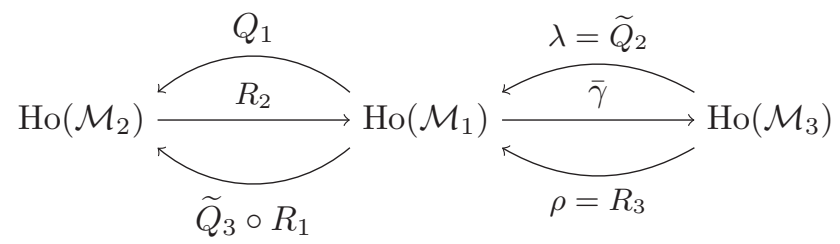

Here, the functor $\bar{\gamma}$ is the quotient functor of Lemma 3.4.

Proof. First we show that the two conditions are equivalent. That is, $\mathcal{W}_{3} \cap \mathcal{F}_{1}=\mathcal{F}_{2}$ and $\mathcal{F}_{3} \subseteq \mathcal{F}_{1}$ if and only if $\widetilde{\mathcal{C}}_{2} \cap \mathcal{W}_{3}=\widetilde{\mathcal{C}}_{1}$ and $\mathcal{F}_{2} \subseteq \mathcal{W}_{3}$. For the "only if" part, the only part that is not clear is $\widetilde{\mathcal{C}}_{2} \cap \mathcal{W}_{3} \subseteq \widetilde{\mathcal{C}}_{1}$. So assume $C \in \widetilde{\mathcal{C}}_{2} \cap \mathcal{W}_{3}$. Use enough projectives of $\left(\widetilde{\mathcal{C}}_{1}, \mathcal{F}_{1}\right)$ to find a short exact sequence $0 \rightarrow F_{1} \rightarrow C_{1} \rightarrow C \rightarrow 0$ with $F_{1} \in \mathcal{F}_{1}$, and $C_{1} \in \widetilde{\mathcal{C}}_{1} \subseteq \mathcal{W}_{3}$. Since $\mathcal{W}_{3}$ is thick we see that $F_{1} \in \mathcal{W}_{3} \cap \mathcal{F}_{1}=\mathcal{F}_{2}$. So with $F_{1} \in \mathcal{F}_{2}$ and $C \in \widetilde{\mathcal{C}}_{2}$, the short exact sequence must split, making $C$ a retract of $C_{1}$. Hence $C$ must be in $\widetilde{\mathcal{C}}_{1}$ finishing the proof of the "only if" part. For the "if" part, the analogous part to show is $\mathcal{W}_{3} \cap \mathcal{F}_{1} \subseteq \mathcal{F}_{2}$. This follows by a similar argument: For $W \in \mathcal{W}_{3} \cap \mathcal{F}_{1}$ start by finding a short exact sequence $0 \rightarrow W \rightarrow F_{2} \rightarrow C_{2} \rightarrow 0$ with $F_{2} \in \mathcal{F}_{2}$, and $C_{2} \in \widetilde{\mathcal{C}}_{2}$. Since $\mathcal{W}_{3}$ is thick we get $C_{2} \in \widetilde{\mathcal{C}}_{2} \cap \mathcal{W}_{3}=\widetilde{\mathcal{C}}_{1}$ and the sequence splits.

Having shown that the two conditions are equivalent, we see at once that they make $\left(\widetilde{\mathcal{C}}_{2}, \mathcal{W}_{3}, \mathcal{F}_{1}\right)$ into a Hovey triple. By the uniqueness of the thick class in a Hovey triple (see $\left[\mathbf{1 1}\right.$, Proposition 3.2]) we conclude that $\mathcal{M}_{1} / \mathcal{M}_{2}=\left(\widetilde{\mathcal{C}_{2}}, \mathcal{W}_{3}, \mathcal{F}_{1}\right)$. It is then easy to see that the identity functor from $\mathcal{M}_{3}$ to $\mathcal{M}_{1} / \mathcal{M}_{2}$ is a right Quillen functor. But since $\mathcal{M}_{3}$ and $\mathcal{M}_{1} / \mathcal{M}_{2}$ both have $\mathcal{W}_{3}$ as its class of trivial objects it follows (see [15, Lemma 5.8] or [11, Lemma 2.7]) that the model structures have the same weak equivalences. So not only is the identity adjunction a Quillen equivalence but we have the equality $\operatorname{Ho}\left(\mathcal{M}_{3}\right)=\operatorname{Ho}\left(\mathcal{M}_{1} / \mathcal{M}_{2}\right)$. 
Note next that $\operatorname{Ho}\left(\mathcal{M}_{2}\right) \cong \operatorname{Ho}\left(\mathcal{M}_{1} / \mathcal{M}_{3}\right)$. The reason is that

$$
\mathcal{M}_{1} / \mathcal{M}_{3}=\left(\widetilde{\mathcal{C}}_{3}, \mathcal{V}, \mathcal{F}_{1}\right)
$$

for some thick class $\mathcal{V}$, and so

$$
\operatorname{Ho}\left(\mathcal{M}_{1} / \mathcal{M}_{3}\right) \cong\left(\widetilde{\mathcal{C}}_{3} \cap \mathcal{F}_{1}\right) / \sim=\left(\mathcal{C} \cap \mathcal{F}_{2}\right) / \sim \cong \operatorname{Ho}\left(\mathcal{M}_{2}\right) .
$$

The homotopy relations $\sim$ agree in the two model structures by [10, Prop. 4.4(5)] since the cores of $\mathcal{M}_{1} / \mathcal{M}_{3}=\left(\widetilde{\mathcal{C}}_{3}, \mathcal{V}, \mathcal{F}_{1}\right)$ and $\mathcal{M}_{2}=\left(\mathcal{C}, \mathcal{W}_{2}, \mathcal{F}_{2}\right)$ coincide. We claim that the identity map $\mathcal{M}_{1} / \mathcal{M}_{3} \rightarrow \mathcal{M}_{2}$ is a (left) Quillen equivalence. First note that it preserves cofibrant and trivially cofibrant objects, and this implies it is a left Quillen functor. To see it is a Quillen equivalence, we must by [14, Definition 1.3.12] show that for all cofibrant $A \in \mathcal{M}_{1} / \mathcal{M}_{3}$ and fibrant $B \in \mathcal{M}_{2}$ argue that a map $f: A \rightarrow B$ is a weak equivalence in $\mathcal{M}_{2}$ if and only if it is a weak equivalence in $\mathcal{M}_{1} / \mathcal{M}_{3}$. So let $f: A \rightarrow B$ be a map with $A \in \widetilde{\mathcal{C}}_{3}$ and $B \in \mathcal{F}_{2}$. Assuming it is a weak equivalence in $\mathcal{M}_{2}$, we may factor it as $f=p i$ where $i: A \rightarrow C$ has cok $i \in \widetilde{\mathcal{C}_{2}}$ and $p: C \rightarrow B$ has $\operatorname{ker} p \in \mathcal{W}_{2} \cap \mathcal{F}_{2}=\mathcal{W}_{3} \cap \mathcal{F}_{3}$. Note then that ker $p \in \mathcal{F}_{3}=\mathcal{V} \cap \mathcal{F}_{1}$ is automatically trivially fibrant in $\mathcal{M}_{1} / \mathcal{M}_{3}$. But since $\mathcal{W}_{3}$ is thick we also have $C \in \mathcal{W}_{3}$, and thus cok $i \in \mathcal{W}_{3}$. So cok $i \in \widetilde{\mathcal{C}}_{2} \cap \mathcal{W}_{3}=\widetilde{\mathcal{C}}_{1}=\widetilde{\mathcal{C}}_{3} \cap \mathcal{V}$. That is, cok $i$ is trivially cofibrant in $\mathcal{M}_{1} / \mathcal{M}_{3}$. Since we have shown $f$ factors as a trivial cofibration followed by a trivial fibration in $\mathcal{M}_{1} / \mathcal{M}_{3}$ we conclude it is also a weak equivalence in $\mathcal{M}_{1} / \mathcal{M}_{3}$. Conversely, if $f$ is a weak equivalence in $\mathcal{M}_{1} / \mathcal{M}_{3}$, the argument reverses and we see that $f$ is a weak equivalence in $\mathcal{M}_{2}$.

Now we wish to construct the recollement. Using that $\mathcal{F}_{2} \subseteq \mathcal{F}_{1}$, we apply Proposition 3.2 to obtain a colocalization sequence which appears as the top horizontal row in the diagram below. However, we also have $\mathcal{F}_{3} \subseteq \mathcal{F}_{1}$ and so we have the analogous colocalization sequence involving $\mathcal{M}_{3}$. But we may rewrite this last colocalization "backwards" so that it appears as a localization sequence. This is the bottom row of the following diagram which also uses the equality $\operatorname{Ho}\left(\mathcal{M}_{3}\right)=\operatorname{Ho}\left(\mathcal{M}_{1} / \mathcal{M}_{2}\right)$ established above:

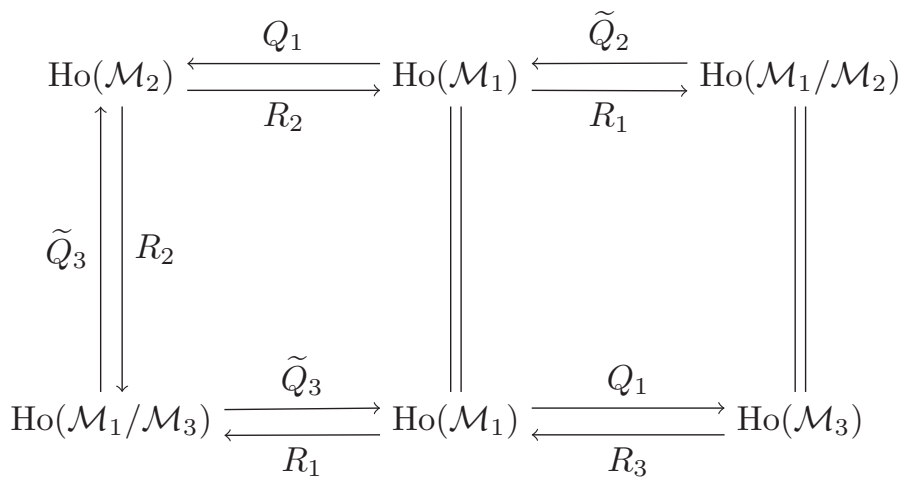

Again, all top functors are left adjoints and all bottom functors are right adjoints. We now let $\bar{\gamma}: \operatorname{Ho}\left(\mathcal{M}_{1}\right) \rightarrow \operatorname{Ho}\left(\mathcal{M}_{3}\right)=\operatorname{Ho}\left(\mathcal{M}_{1} / \mathcal{M}_{2}\right)$ be the quotient functor of Lemma 3.4. We claim, that as functors $\operatorname{Ho}\left(\mathcal{M}_{1}\right)$ to $\operatorname{Ho}\left(\mathcal{M}_{1} / \mathcal{M}_{2}\right)$, there is a natural isomorphism of functors $\left\{j_{A}\right\}: \bar{\gamma} \cong R_{1}$. Indeed given $f: A \rightarrow B$, recall the functor $R_{1}$ works by applying enough injectives using the cotorsion pair $\left(\widetilde{\mathcal{C}}_{1}, \mathcal{F}_{1}\right)$. That is, $R_{1}$ acts by $f \mapsto \hat{f}$ 
where $\hat{f}$ is any map making the diagram below commute. Here, the rows are exact and $F_{1}, F_{1}^{\prime} \in \mathcal{F}_{1}$ and $C_{1}, C_{1}^{\prime} \in \widetilde{\mathcal{C}}_{1}$.

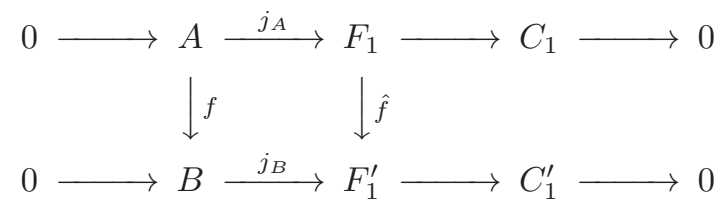

Since $\widetilde{\mathcal{C}_{1}}=\mathcal{C} \cap \mathcal{W}_{1}={ }^{\perp} \mathcal{F}_{1}=\widetilde{\mathcal{C}}_{2} \cap \mathcal{W}_{3}$, we see in particular that $C_{1}, C_{1}^{\prime} \in \mathcal{W}_{3}$. That is, they are trivial in $\mathcal{M}_{1} / \mathcal{M}_{2}$ which means the maps $\left\{j_{A}\right\}$ are providing a natural isomorphism $\left\{j_{A}\right\}: \bar{\gamma} \cong R_{1}$.

Similarly we get that the functors $\bar{\gamma}, Q_{1}: \operatorname{Ho}\left(\mathcal{M}_{1}\right) \rightarrow \operatorname{Ho}\left(\mathcal{M}_{3}\right)$ admit a natural isomorphism $\left\{p_{A}\right\}: Q_{1} \cong \bar{\gamma}$. To see this, recall the functor $Q_{1}$ works by using enough projectives of the cotorsion pair $\left(\mathcal{C}, \widetilde{\mathcal{F}}_{1}\right)$. So for a given $f: A \rightarrow B$ we construct a diagram as below with exact rows and $F_{1}, F_{1}^{\prime} \in \widetilde{\mathcal{F}}_{1}$ and $C_{1}, C_{1}^{\prime} \in \mathcal{C}$.

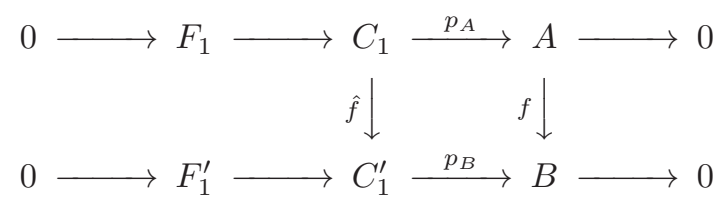

But $F_{1}, F_{1}^{\prime} \in \widetilde{\mathcal{F}}_{1}=\mathcal{W}_{1} \cap \mathcal{F}_{1}=\mathcal{C}^{\perp}=\mathcal{W}_{3} \cap \mathcal{F}_{3}$, so again, $F_{1}, F_{1}^{\prime}$ are trivial in $\mathcal{M}_{3}$. This shows the maps $\left\{p_{A}\right\}$ are providing a natural isomorphism $\left\{p_{A}\right\}: Q_{1} \cong \bar{\gamma}$.

Going back to $(*)$ one can easily check that using the natural isomorphism $\left\{j_{A}\right\}$ : $\bar{\gamma} \cong R_{1}$, we may simply replace $R_{1}$ by $\bar{\gamma}$ and the top row will still be a colocalization sequence. Similarly, using the natural isomorphism $\left\{p_{A}\right\}: Q_{1} \cong \bar{\gamma}$ we may replace $Q_{1}$ with $\bar{\gamma}$ and the bottom row will remain a localization sequence. That is, the right square of $(*)$ is glued along $\bar{\gamma}$, and becoming

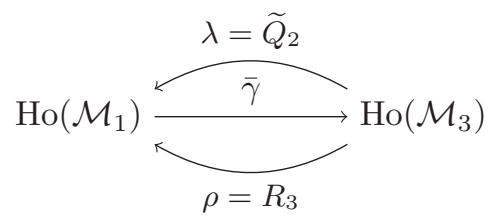

We now turn to the left square of the diagram $(*)$. We claim the functor $\operatorname{Ho}\left(\mathcal{M}_{2}\right) \stackrel{R_{2}}{\longrightarrow}$ $\operatorname{Ho}\left(\mathcal{M}_{1}\right)$ is isomorphic to the composite $\operatorname{Ho}\left(\mathcal{M}_{2}\right) \stackrel{R_{2}}{\longrightarrow} \operatorname{Ho}\left(\mathcal{M}_{1} / \mathcal{M}_{3}\right) \stackrel{\widetilde{Q}_{3}}{\longrightarrow} \operatorname{Ho}\left(\mathcal{M}_{1}\right)$. Indeed, $R_{2}$ acts by $f \mapsto \hat{f}$ where $\hat{f}$ is any map making the diagram below commute, where $F_{2}, F_{2}^{\prime} \in \mathcal{F}_{2}$ and $C_{2}, C_{2}^{\prime} \in \widetilde{\mathcal{C}_{2}}$.

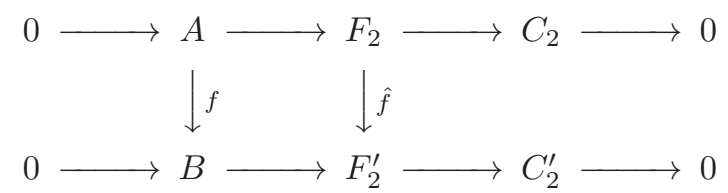

Now applying $\operatorname{Ho}\left(\mathcal{M}_{1} / \mathcal{M}_{3}\right) \stackrel{\widetilde{Q}_{3}}{\longrightarrow} \operatorname{Ho}\left(\mathcal{M}_{1}\right)$ to $\hat{f}$ gives us $\bar{f}$ in the next diagram, where 


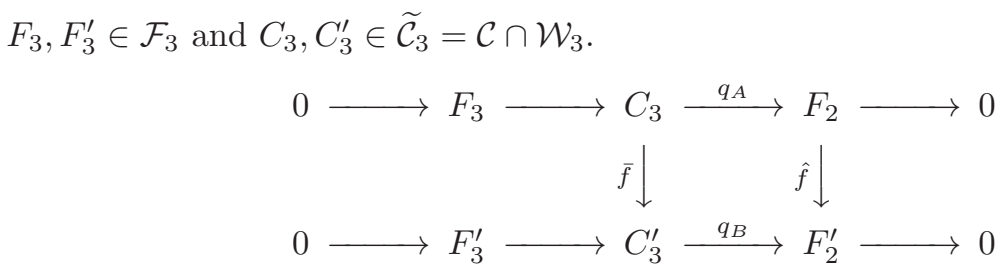

But since $F_{2}, F_{2}^{\prime} \in \mathcal{F}_{2}=\mathcal{W}_{3} \cap \mathcal{F}_{1}$ and $\mathcal{W}_{3}$ is thick, we see that $F_{3}, F_{3}^{\prime} \in \mathcal{W}_{3} \cap \mathcal{F}_{3}=$ $\mathcal{W}_{1} \cap \mathcal{F}_{1}$. That is, $F_{3}, F_{3}^{\prime}$ are trivial in $\mathcal{M}_{1}$ and hence the maps $\left\{q_{A}\right\}$ are providing a natural isomorphism $\left\{q_{A}\right\}: \widetilde{Q}_{3} \circ R_{2} \cong R_{2}$.

Finally, recall that we have shown the vertical functors in the diagram $(*)$ to be equivalences. So each of these functors are both right and left adjoints of each other. So the isomorphism $\left\{q_{A}\right\}: \widetilde{Q}_{3} \circ R_{2} \cong R_{2}$ means that the functor $\operatorname{Ho}\left(\mathcal{M}_{2}\right) \stackrel{R_{2}}{\longrightarrow} \operatorname{Ho}\left(\mathcal{M}_{1}\right)$ has right adjoint the composite $\operatorname{Ho}\left(\mathcal{M}_{1}\right) \stackrel{R_{1}}{\longrightarrow} \operatorname{Ho}\left(\mathcal{M}_{1} / \mathcal{M}_{3}\right) \stackrel{\widetilde{Q}_{3}}{\longrightarrow} \operatorname{Ho}\left(\mathcal{M}_{2}\right)$.

We have the dual notion of left localization, denoted $\mathcal{M}_{2} \backslash \mathcal{M}_{1}$, and the dual statements of the above. In particular we have the following left recollement theorem.

Theorem 3.6 (Left recollement theorem). Let $\mathcal{A}$ be an abelian category with three hereditary model structures, as below, whose cores all coincide:

$$
\mathcal{M}_{1}=\left(\mathcal{C}_{1}, \mathcal{W}_{1}, \mathcal{F}\right), \quad \mathcal{M}_{2}=\left(\mathcal{C}_{2}, \mathcal{W}_{2}, \mathcal{F}\right), \quad \mathcal{M}_{3}=\left(\mathcal{C}_{3}, \mathcal{W}_{3}, \mathcal{F}\right)
$$

If $\mathcal{W}_{3} \cap \mathcal{C}_{1}=\mathcal{C}_{2}$ and $\mathcal{C}_{3} \subseteq \mathcal{C}_{1}$ (or equivalently, $\widetilde{\mathcal{F}}_{2} \cap \mathcal{W}_{3}=\widetilde{\mathcal{F}}_{1}$ and $\mathcal{C}_{2} \subseteq \mathcal{W}_{3}$ ), then $\mathcal{M}_{2} \backslash \mathcal{M}_{1}$ is Quillen equivalent to $\mathcal{M}_{3}$ and $\mathcal{M}_{3} \backslash \mathcal{M}_{1}$ is Quillen equivalent to $\mathcal{M}_{2}$. In fact, we have a recollement as shown below.

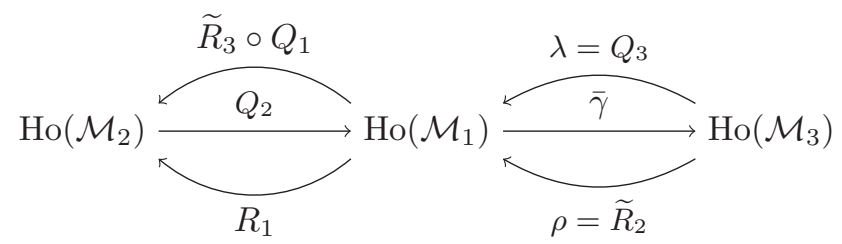

Here, the functor $\bar{\gamma}$ is the quotient functor of (the dual of) Lemma 3.4.

\section{Models for mock homotopy categories of projectives}

As described in the introduction we will now give an interesting application of Theorem 3.6. In Theorem 4.8 we present three interrelated Hovey triples which immediately yield the recollement of Murfet from [17]. We see that the recollement not only holds for many non-noetherian schemes $X$, but we give a vast generalization of the idea in Theorem 4.10.

Let $(\mathcal{F}, \mathcal{C})$ denote the flat cotorsion pair in the category $\mathrm{Q} \operatorname{co}(X)$ of quasi-coherent sheaves on $X$, where $X$ is any scheme in which $\mathrm{Qco}(X)$ has a flat generator. This is true for example when $X$ is a quasi-compact semi-separated scheme. Then we already have from [9, Theorem 5.5 and Section 5.3], the following two complete hereditary (and indeed small in the sense of $[\mathbf{1 5}$, Section 6$]$ ) cotorsion pairs in the category 
$\mathrm{Ch}(\mathrm{Qco}(X))$ of chain complexes of quasi-coherent sheaves:

$$
\left(d w \tilde{\mathcal{F}}, d w \widetilde{\mathcal{F}}^{\perp}\right) \quad \text { and } \quad\left(e x \widetilde{\mathcal{F}}, e x \widetilde{\mathcal{F}}^{\perp}\right) .
$$

Here, $d w \widetilde{\mathcal{F}}$ is the class of all complexes $X$ with each $X_{n} \in \mathcal{F}$, while $e x \widetilde{\mathcal{F}}$ is the class of all exact complexes $X$ with each $X_{n} \in \mathcal{F}$. Using the characterizations of $d w \widetilde{\mathcal{F}}^{\perp}$ and $e x \widetilde{\mathcal{F}}^{\perp}$ from [9, Propositions 3.2 and 3.3] we can easily argue that each of the above cotorsion pairs has core equal to the class of all contractible complexes with components in $\mathcal{F} \cap \mathcal{C}$. We also have the cotorsion pairs $(d g \widetilde{\mathcal{F}}, \widetilde{\mathcal{C}})$ and $(\widetilde{\mathcal{F}}, d g \widetilde{\mathcal{C}})$ from $[7]$ and $[\mathbf{8}]$ which have the same cores. Here, we note in particular that $\widetilde{\mathcal{F}}$ is the class of all exact complexes of flat quasi-coherent sheaves with flat cycles. The following are immediate corollaries of [13, Theorem 1.1].

Corollary 4.1. There is an hereditary abelian model structure

$$
\mathcal{M}_{1}=\left(d w \widetilde{\mathcal{F}}, \mathcal{W}_{1}, d g \widetilde{\mathcal{C}}\right)
$$

whose core equals the class of all contractible complexes with components in $\mathcal{F} \cap \mathcal{C}$.

Proof. Apply [13, Theorem 1.1] to the cotorsion pairs $\left(d w \widetilde{\mathcal{F}}, d w \widetilde{\mathcal{F}}^{\perp}\right)$ and $(\widetilde{\mathcal{F}}, d g \widetilde{\mathcal{C}})$ to obtain the thick class $\mathcal{W}_{1}$ yielding the Hovey triple.

Remark 4.2. The homotopy category $\operatorname{Ho}\left(\mathcal{M}_{1}\right)$ is equivalent to Murfet's mock homotopy category of projectives, $K_{m}(\operatorname{Proj} X)$. To see this, lets recall the original definition of $K_{m}(\operatorname{Proj} X)$. First, let $K($ Flat $X)$ be the chain homotopy category of all complexes of flat quasi-coherent sheaves. It is a triangulated category with coproducts. By definition, $K_{m}(\operatorname{Proj} X)$ is the Verdier quotient of $K($ Flat $X)$ by the localizing subcategory consisting of all exact complexes with flat cycles [17, Def. 3.3/Prop. 3.4]. In our notation, these are exactly the complexes in $\widetilde{\mathcal{F}}$. The model structure $\mathcal{M}_{1}=$ $\left(d w \widetilde{\mathcal{F}}, \mathcal{W}_{1}, d g \widetilde{\mathcal{C}}\right)$ has been constructed so that $d w \widetilde{\mathcal{F}} \cap \mathcal{W}_{1}=\widetilde{\mathcal{F}}$. In other words, a complex of flats is zero in $\operatorname{Ho}\left(\mathcal{M}_{1}\right)$ if and only if it lies in $\widetilde{\mathcal{F}}$. In fact, by $[\mathbf{1 0}$, Prop. 5.2/Cor. 5.4], any hereditary abelian model structure restricts to a canonically equivalent (exact) model structure on the full subcategory of all cofibrant objects. In the current case, it means we immediately get a Hovey triple, $(d w \widetilde{\mathcal{F}}, \widetilde{\mathcal{F}}, d w \widetilde{\mathcal{F}} \cap d g \widetilde{\mathcal{C}})$, on the exact category $\mathrm{Ch}(F$ lat $X)$ of all chain complexes of flat quasi-coherent sheaves.

In the affine case $X=\operatorname{Spec} R$, it is fundamental that $K_{m}(\operatorname{Proj} X) \cong K(\operatorname{Proj} X)$, the chain homotopy category of all complexes of projectives. This also has a model category explanation as follows. From [4, Corollary 6.4], we see that $K(\operatorname{Proj} X)$ is the homotopy category of an hereditary abelian model structure $\left(d w \widetilde{\mathcal{P}}, d w \widetilde{\mathcal{P}}^{\perp}, \mathcal{A}\right)$ on $\mathrm{Ch}(R)$, where $d w \widetilde{\mathcal{P}}$ is the class of all complexes of projective modules, and $\mathcal{A}$ is the class of all chain complexes. Following [19], the complexes in $d w \widetilde{\mathcal{P}}^{\perp}$ are called contraacyclic. Turning back to $\mathcal{M}_{1}=\left(d w \widetilde{\mathcal{F}}, \mathcal{W}_{1}, d g \widetilde{\mathcal{C}}\right)$, it turns out that $\mathcal{W}_{1}$ is precisely the class of contraacyclic complexes; that is, $\mathcal{W}_{1}=d w \widetilde{\mathcal{P}}^{\perp}$. (Reason) $d w \widetilde{\mathcal{P}}^{\perp}$ is thick and Neeman has shown in $\left[\mathbf{1 8}\right.$, Theorem 8.6] that $\widetilde{\mathcal{F}}=d w \widetilde{\mathcal{F}} \cap d w \widetilde{\mathcal{P}}^{\perp}$. Lemma 4.3 below now implies that $\left(d w \widetilde{\mathcal{F}}, d w \widetilde{\mathcal{P}}^{\perp}, d g \widetilde{\mathcal{C}}\right)$ is a Hovey triple. But the uniqueness of the class of trivial objects in any Hovey triple implies $\mathcal{W}_{1}=d w \widetilde{\mathcal{P}}^{\perp}$.

Lemma 4.3. Suppose we have two complete cotorsion pairs $(\mathcal{Q}, \widetilde{\mathcal{R}})$ and $(\widetilde{\mathcal{Q}}, \mathcal{R})$ in an abelian category and that we also have a thick class $\mathcal{W}$. Then the following hold: 
1. If $\widetilde{\mathcal{Q}}=\mathcal{Q} \cap \mathcal{W}$ and $\widetilde{\mathcal{R}} \subseteq \mathcal{W}$, then also $\widetilde{\mathcal{R}}=\mathcal{W} \cap \mathcal{R}$. That is, $(\mathcal{Q}, \mathcal{W}, \mathcal{R})$ is a Hovey triple.

2. If $\widetilde{\mathcal{R}}=\mathcal{W} \cap \mathcal{R}$ and $\widetilde{\mathcal{Q}} \subseteq \mathcal{W}$, then also $\widetilde{\mathcal{Q}}=\mathcal{Q} \cap \mathcal{W}$. That is, $(\mathcal{Q}, \mathcal{W}, \mathcal{R})$ is a Hovey triple.

Proof. The statements are proved similarly, and we will prove (1). We have by assumption that $\widetilde{\mathcal{Q}} \subseteq \mathcal{Q}$ and consequently $\widetilde{\mathcal{R}} \subseteq \mathcal{R}$. We are also assuming $\widetilde{\mathcal{R}} \subseteq \mathcal{W}$, and so we have $\widetilde{\mathcal{R}} \subseteq \mathcal{W} \cap \mathcal{R}$.

It is left to show $\widetilde{\mathcal{R}} \supseteq \mathcal{W} \cap \mathcal{R}$. Letting $X \in \mathcal{W} \cap \mathcal{R}$, we use completeness of the cotorsion pair $(\mathcal{Q}, \widetilde{\mathcal{R}})$ to find a short exact sequence $0 \rightarrow X \rightarrow R \rightarrow Q \rightarrow 0$ with $R \in$ $\widetilde{\mathcal{R}}$ and $Q \in \mathcal{Q}$. We see that this forces $Q \in \mathcal{Q} \cap \mathcal{W}=\widetilde{\mathcal{Q}}$. Hence the sequence must split, forcing $X$ to be a retract of an object in $\widetilde{\mathcal{R}}$. So $X$ is also in $\widetilde{\mathcal{R}}$.

Corollary 4.4. There is an hereditary abelian model structure

$$
\mathcal{M}_{2}=\left(e x \widetilde{\mathcal{F}}, \mathcal{W}_{2}, d g \widetilde{\mathcal{C}}\right)
$$

whose core equals the class of all contractible complexes with components in $\mathcal{F} \cap \mathcal{C}$.

Proof. Apply [13, Theorem 1.1] to the cotorsion pairs $\left(e x \widetilde{\mathcal{F}}, e x \widetilde{\mathcal{F}}^{\perp}\right)$ and $(\widetilde{\mathcal{F}}, d g \widetilde{\mathcal{C}})$ to obtain the thick class $\mathcal{W}_{2}$ yielding the Hovey triple.

Remark 4.5. The homotopy category $\operatorname{Ho}\left(\mathcal{M}_{2}\right)$ is equivalent to Murfet's mock projective stable derived category, $K_{m, a c}(\operatorname{Proj} X)$. By definition, it is the full subcategory of $K_{m}(\operatorname{Proj} X)$ consisting of all exact complexes. In the affine case $X=$ Spec $R$, the fundamental fact is that $K_{m, a c}(\operatorname{Proj} X) \cong K_{a c}(\operatorname{Proj} X)$, the chain homotopy category of all exact (acyclic) complexes of projectives. For a general ring $R$, this is called the projective stable derived category, and a model was constructed for it in $[\mathbf{4}$, Corollary 6.5]. It is the hereditary abelian model structure $\left(\right.$ ex $\widetilde{\mathcal{P}}$, ex $\left.\widetilde{\mathcal{P}}^{\perp}, \mathcal{A}\right)$ on $\operatorname{Ch}(R)$ where ex $\widetilde{\mathcal{P}}$ is the class of all exact complexes of projective modules, and $\mathcal{A}$ is the class of all chain complexes. Interpreting Corollary 4.4 in the affine case $X=\operatorname{Spec} R$, we do in fact have the desired equality $\mathcal{W}_{2}=e x \widetilde{\mathcal{P}}^{\perp}$. (Reason) Similar to the remark following Corollary 4.1, it will follow from Lemma 4.3 by showing $\widetilde{\mathcal{F}}=e x \widetilde{\mathcal{F}} \cap e x \widetilde{\mathcal{P}}^{\perp}$. But letting $\mathcal{E}$ denote the class of all exact complexes we have

$$
\text { ex } \widetilde{\mathcal{F}} \cap e x \widetilde{\mathcal{P}}^{\perp}=(d w \widetilde{\mathcal{F}} \cap \mathcal{E}) \cap e x \widetilde{\mathcal{P}}^{\perp}=d w \widetilde{\mathcal{F}} \cap\left(\mathcal{E} \cap e x \widetilde{\mathcal{P}}^{\perp}\right)=d w \widetilde{\mathcal{F}} \cap d w \widetilde{\mathcal{P}}^{\perp}=\widetilde{\mathcal{F}}
$$

The last equality is again using Neeman's result, but the second to last equality also requires justification. To see $\mathcal{E} \cap e x \widetilde{\mathcal{P}}^{\perp}=d w \widetilde{\mathcal{P}}^{\perp}$ we use yet another application of Lemma 4.3. Indeed, as shown in [4], we know that $\left(d w \widetilde{\mathcal{P}}, d w \widetilde{\mathcal{P}}^{\perp}\right)$ and $\left(e x \widetilde{\mathcal{P}}, e x \widetilde{\mathcal{P}}^{\perp}\right)$ are each complete cotorsion pairs and of course $\mathcal{E}$ is thick.

Corollary 4.6. Let $\mathcal{E}$ denote the class of all exact complexes. There is an hereditary abelian model structure

$$
\mathcal{M}_{3}=(d g \widetilde{\mathcal{F}}, \mathcal{E}, d g \widetilde{\mathcal{C}}),
$$

whose core equals the class of all contractible complexes with components in $\mathcal{F} \cap \mathcal{C}$.

Proof. This is the flat model structure from $[\mathbf{7}]$ and $[\mathbf{8}]$ corresponding to $(d g \widetilde{\mathcal{F}}, \widetilde{\mathcal{C}})$ and $(\widetilde{\mathcal{F}}, d g \widetilde{\mathcal{C}})$. 
Remark 4.7. $\mathcal{M}_{3}$ is a model for $\mathcal{D}(X)$, the derived category of the scheme $X$. This follows from the fact that the trivial objects are precisely the exact complexes. In the affine case we do indeed have $\mathcal{E}=d g \widetilde{\mathcal{P}}^{\perp}$, where $d g \widetilde{\mathcal{P}}$ is the class of all DG-projective complexes.

Theorem 4.8. Let $X$ be any scheme in which $\mathrm{Qco}(X)$ has a flat generator. Then $\mathcal{M}_{1}, \mathcal{M}_{2}, \mathcal{M}_{3}$ above satisfy the hypotheses of Theorem 3.6 and recover the recollement of Murfet.

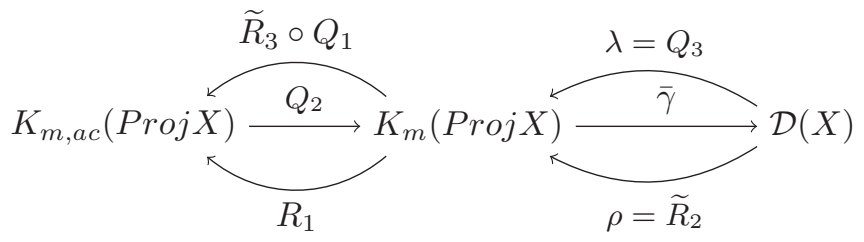

\subsection{Generalizing to other cotorsion pairs}

Rather than Qco $(X)$, one may instead wish to consider the flat cotorsion pair $(\mathcal{F}, \mathcal{C})$ in the category of sheaves of $\mathcal{O}$-modules where $\mathcal{O}=\left(\mathcal{O}_{X}, X\right)$ is a ringed space. In fact, one may wish to start with other Grothendieck categories and cotorsion pairs other than the flat one.

So let $\mathcal{G}$ be any Grothendieck category and let $(\mathcal{A}, \mathcal{B})$ be a complete hereditary cotorsion pair. Then from [9, Proposition 3.2 and 3.3] we have the first two cotorsion pairs, and from [7, Proposition 3.6] we have the second two cotorsion pairs:

$$
\left(d w \widetilde{\mathcal{A}}, d w \widetilde{\mathcal{A}}^{\perp}\right), \quad\left(\operatorname{ex} \widetilde{\mathcal{A}}, e x \widetilde{\mathcal{A}}^{\perp}\right), \quad(d g \widetilde{\mathcal{A}}, \widetilde{\mathcal{B}}), \quad(\widetilde{\mathcal{A}}, d g \widetilde{\mathcal{B}}) .
$$

The definitions are analogous to those above using the flat cotorsion pair $(\mathcal{F}, \mathcal{C})$. They are all hereditary since $(\mathcal{A}, \mathcal{B})$ is assumed to be. Moreover, just as in the flat situation, we can easily argue that each of the above cotorsion pairs has core equal to the class of all contractible complexes with components in $\mathcal{A} \cap \mathcal{B}$.

Now assume $(\mathcal{A}, \mathcal{B})$ is cogenerated by a set $\mathcal{S}$ and that $\mathcal{A}$ contains a generating set $\left\{U_{i}\right\}$ for $\mathcal{G}$. The methods of $[\mathbf{9}],[\mathbf{7}$, Proposition 3.6], and [8] proving completeness of the four associated cotorsion pairs above rely on the extra assumption that $\mathcal{A}$ be closed under direct limits. Others have since removed this assumption, giving us the following lemma.

Lemma 4.9. Let $\mathcal{G}$ be any Grothendieck category and $(\mathcal{A}, \mathcal{B})$ an hereditary cotorsion pair cogenerated by some set $\mathcal{S}$ and such that $\mathcal{A}$ contains a generating set $\left\{U_{i}\right\}$ for $\mathcal{G}$. Then the four induced cotorsion pairs on $\mathrm{Ch}(\mathcal{G})$ listed above are also cogenerated by a set and complete cotorsion pairs.

Proof. We note that in the case of a concrete Grothendieck category, Theorem 3.1 of [6] immediately gives us the result. For a general Grothendieck category we can argue as follows. First, note that $\left\{D^{n}\left(U_{i}\right)\right\}$ is a generating set for $\operatorname{Ch}(\mathcal{G})$ and is contained in each of the four classes $d w \widetilde{\mathcal{A}}$, ex $\widetilde{\mathcal{A}}, d g \widetilde{\mathcal{A}}$, and $\widetilde{\mathcal{A}}$. By Proposition 2.4 we see that $\mathcal{A}$ is deconstructible and that it is enough to show each of these four classes is also deconstructible. But Štovíček has shown in [21, Theorem 4.2] that since $\mathcal{A}$ is deconstructible we automatically must have that $d w \widetilde{\mathcal{A}}, d g \widetilde{\mathcal{A}}$, and $\widetilde{\mathcal{A}}$ are 
each deconstructible. This proves the lemma for three of the four cotorsion pairs but we still must deal with ex $\widetilde{\mathcal{A}}$. Here we note that ex $\widetilde{\mathcal{A}}=d w \widetilde{\mathcal{A}} \cap \mathcal{E}$ where $\mathcal{E}$ is the class of all exact complexes. However, in any Grothendieck category $\mathcal{G}$, the class of all objects is itself deconstructible; for example, see [22, Proposition 3.13]. From this it follows from $[\mathbf{2 1}$, Theorem $4.2(2)]$ that $\mathcal{E}$ is also deconstructible. We now turn around and conclude that the intersection of the two deconstructible classes ex $\widetilde{\mathcal{A}}=d w \widetilde{\mathcal{A}} \cap \mathcal{E}$ is also deconstructible by [21, Proposition 2.9(2)].

It now follows from the above that Corollaries 4.1, 4.4, and 4.6 all have direct generalizations from the flat cotorsion pair $(\mathcal{F}, \mathcal{C})$ to more general hereditary cotorsion pairs $(\mathcal{A}, \mathcal{B})$. This leads us to the following generalization of Theorem 4.8 .

Theorem 4.10. Let $\mathcal{G}$ be any Grothendieck category and $(\mathcal{A}, \mathcal{B})$ an hereditary cotorsion pair cogenerated by some set $\mathcal{S}$ and such that $\mathcal{A}$ contains a generating set $\left\{U_{i}\right\}$ for $\mathcal{G}$. Then we have three hereditary abelian model structure:

$$
\mathcal{M}_{1}=\left(d w \widetilde{\mathcal{A}}, \mathcal{W}_{1}, d g \widetilde{\mathcal{B}}\right), \quad \mathcal{M}_{2}=\left(e x \widetilde{\mathcal{A}}, \mathcal{W}_{2}, d g \widetilde{\mathcal{B}}\right), \quad \mathcal{M}_{3}=(d g \widetilde{\mathcal{A}}, \mathcal{E}, d g \widetilde{\mathcal{B}}) .
$$

They satisfy that each has core equaling the class of all contractible complexes with components in $\mathcal{A} \cap \mathcal{B}$ and so Theorem 3.6 applies to yield a recollement.

\section{References}

[1] H. Becker, Models for singularity categories, Adv. Math. vol. 254, 2014, pp. 187232.

[2] A.A. Beilinson, J. Bernstein, and P. Deligne, Faisceaux pervers, Astérisque, vol. 100, 1982.

[3] D. Bravo, The stable derived category of a ring via model categories, Ph.D. thesis, Wesleyan University, May 2011.

[4] D. Bravo, J. Gillespie, and M. Hovey, The stable module category of a general ring, arXiv:1405.5768.

[5] E. Enochs and O. Jenda, Relative homological algebra, De Gruyter Exp. Math. no. 30, Walter De Gruyter, New York, 2000.

[6] E. Enochs, S. Estrada, and A. Iacob, Cotorsion pairs, model structures and adjoints in homotopy categories, Houston J. Math. vol. 40, no. 1, 2014, pp. 4361 .

[7] J. Gillespie, The flat model structure on $C h(R)$, Trans. Amer. Math. Soc. vol. 356, no. 8, 2004, pp. 3369-3390.

[8] J. Gillespie, Kaplansky classes and derived categories, Math. Z. vol. 257, no. 4, 2007, pp. 811-843.

[9] J. Gillespie, Cotorsion pairs and degreewise homological model structures, Homology, Homotopy Appl. vol. 10, no. 1, 2008, pp. 283-304.

[10] J. Gillespie, Model structures on exact categories, J. Pure Appl. Algebra vol. 215, 2011, pp. 2892-2902.

[11] J. Gillespie, Gorenstein complexes and recollements from cotorsion pairs, Adv. Math. vol. 291, 2016, pp. 859-911. 
[12] J. Gillespie, Exact model structures and recollements, J. Algebra vol. 458, 2016, pp. 265-306.

[13] J. Gillespie, How to construct a Hovey triple from two cotorsion pairs, Fund. Math. vol. 230, no. 3, 2015, pp. 281-289.

[14] M. Hovey, Model categories, Math. Surv. Monogr. vol. 63, American Mathematical Society, 1999.

[15] M. Hovey, Cotorsion pairs, model category structures, and representation theory, Math. Z. vol. 241, 2002, pp. 553-592.

[16] H. Krause, The stable derived category of a Noetherian scheme, Compos. Math. vol. 141, no. 5, 2005, pp. 1128-1162.

[17] D. Murfet, The mock homotopy category of projectives and Grothendieck duality, Ph.D. thesis, Australian National University, 2007 (online at www.therisingsea.org).

[18] A. Neeman, The homotopy category of flat modules, and Grothendieck duality, Invent. Math. vol. 174, no. 2, 2008, pp. 255-308.

[19] L. Positselski, Two kinds of derived categories, Koszul duality, and comodulecontramodule correspondence, Mem. Amer. Math. Soc. vol. 212, no. 996, 2011, arXiv:0905.2621.

[20] M. Saorín and J. Šťovíček, On exact categories and applications to triangulated adjoints and model structures, Adv. Math. vol. 228, no. 2, 2011, pp. 968-1007.

[21] J. Štovíček, Deconstructibility and the Hill lemma in Grothendieck categories, Forum Math. vol. 25, no. 1, 2013, pp. 193-219.

[22] J. Štovíček, Exact model categories, approximation theory, and cohomology of quasi-coherent sheaves, Advances in Representation Theory of Algebras (ICRA Bielefeld, Germany, 8-17 August 2012), EMS Series of Congress Reports, European Mathematical Society Publishing House, 2014, pp. 297-367.

James Gillespie jgillesp@ramapo.edu

Ramapo College of New Jersey, School of Theoretical and Applied Science, 505 Ramapo Valley Road, Mahwah, NJ 07430, USA 